\title{
Identification of Floral Scent Profiles in Bearded Irises
}

\author{
Yuan Yuan ${ }^{1}$, Ye Sun ${ }^{1}$, Yanchun Zhao ${ }^{2}$, Chungui Liu ${ }^{1}$, Xiulan Chen ${ }^{1}$, Fengtong $\mathrm{Li}^{1}{ }^{1} * \mathbb{D}$ and \\ Jianzhong Bao 1,* \\ 1 Institute of Agricultural Sciences for Lixiahe Region in Jiangsu, Yangzhou 225007, Jiangsu Province, China; \\ ocean1209@126.com (Y.Y.); sunye9999@126.com (Y.S.); horlcg@sina.com (C.L.); yzchxl@163.com (X.C.) \\ 2 Civil Engineering Department, Yangzhou Polytechnic College, Yangzhou 225009, Jiangsu Province, China; \\ zhaoyc083@163.com \\ * Correspondence: 1ftchina@163.com (F.L.); yzbjz@126.com (J.B.); Tel.: +86-514-8730-2156 (F.L.)
}

Received: 10 April 2019; Accepted: 1 May 2019; Published: 7 May 2019

\begin{abstract}
Bearded irises are ornamental plants with distinctive floral fragrance grown worldwide. To identify the floral scent profiles, twenty-seven accessions derived from three bearded iris, including Iris. germanica, I. pumila and I. pallida were used to investigate the composition and relative contents of floral scent components by headspace solid-phase microextraction (HS-SPME) and gas chromatography-mass spectrometry (GC-MS). A total of 219 floral scent components were detected in blooming flowers. The scent profile varied significantly among and within the three investigated species. Principal component analysis (PCA) indicated that terpenes, alcohols and esters contributed the most to the floral scent components and 1-caryophyllene, linalool, citronellol, methyl cinnamate, $\beta$-cedrene, thujopsene, methyl myristate, linalyl acetate, isosafrole, nerol, geraniol were identified as the major components. In a hierarchical cluster analysis, twenty-seven accessions could be clustered into six different groups, most of which had representative scent components such as linalool, citronellyl acetate, thujopsene, citronellol, methyl cinnamate and 1-caryophyllene. Our findings provide a theoretical reference for floral scent evaluation and breeding of bearded irises.
\end{abstract}

Keywords: bearded iris; floral scent component; GC-MS; SPME

\section{Introduction}

Floral scent is a complex mixture of low molecular weight volatile secondary metabolite compounds released by plant flowers, which gives the flower its unique fragrance Floral scent not only plays an important role in plants' reproductive process by attracting pollinators [1], but also enhances the aesthetic properties of ornamental plants [2]. Detailed studies on floral scent profiles have been carried out on many traditional aromatic plants, including orchids [3,4], Osmanthus fragrans [5], tree peony [6], Lilium [7], rose [8,9], Prunus mume [10], Chimonanthus praecox [11] and so forth. However, in recent years, ornamental plant researchers and breeders have paid more and more attention on some novel fragrant ornamental plants such as Luculia pinceana [12], Hosta [13], Lagerstroemia indica [14], whose floral fragrances are distinctive, so as to discover new floral scent components which could be applied in generating novel varieties.

Bearded iris is an Iris (Iridaceae) member that gains its name from the line of thick hairs that emerges from the throat of the flowers. These hairs form a long, furry caterpillar towards the back of the fall petals so as to guide pollinators towards the pollen [15]. The colors of the hairs could present different morphs among cultivars or species. Bearded irises are popular ornamental plants worldwide, widely used in gardens and flower borders, as potted plants, an even cut flowers and other production fields. In addition to the visible characteristics, bearded iris is one of the few groups in the Iris genus which produces a pleasant fragrance. As a result, bearded irises have a particularly high ornamental value in both the visual and olfactory sense. I. germanica has the greatest number of 
cultivated varieties in the bearded iris group. During the long evolution history of I. germanica, more than eight related species were introduced into hybridization breeding, which greatly improved the flower type, flower color, plant height, leaf color and floral fragrance ornamental traits of I. germanica varieties. Precisely because of this, I. germanica has become the most popular iris in garden use. So far, although great progress has been made in the studies on cultivation [16,17], propagation [18,19], genetics and breeding [20-23], chemical characterizations of the floral scent in I. germanica and other species of bearded iris have not been reported to date, which hinders the development and utilization for the varieties with different fragrances.

In this work, we explore the floral scent profile variations of bearded irises, mainly in I. germanica wild species and cultivars (twenty-three accessions), as well as in I. germanica's two related species I. pallida (two accessions) and I. pumila (two accessions) through headspace solid-phase microextraction coupled with gas chromatography-mass spectrometry. Firstly, we identify the specific floral scent volatile compounds in the three investigated bearded iris species and compare the scent compositions in the twenty-seven accessions with the expectation of encountering species-specific and/or cultivars-specific volatile components. Secondly, we clarify the most contributing components in the investigated bearded irises based on the scent component profiles through principle components analysis. Finally, we classify the twenty-seven accessions into different floral fragrance patterns according to their floral scent components by using hierarchical cluster analysis to clarify the origin of the distinctive fragrance of the flowers. The results would not only provide a theoretical reference for elucidation of the chemical mechanism of floral fragrance formation in bearded irises, but also lay a foundation for the fragrant varieties breeding in the future.

\section{Results and Discussion}

\subsection{Identification of Floral Scent Components in Bearded Iris Accessions}

The total ion chromatogram of scent components emitted from the flowers of twenty-seven accessions of three bearded iris species was shown in Figure S1. A total of 219 scent components in 10 categories including 42 terpenes, 19 alkanes, 11 aromatic compounds, 52 esters, 41 alcohols, 17 ketones, 21 aldehydes, nine ethers, four phenols, and three acids were identified by comparison with a known database (Tables 1 and 2). The number of volatile components varied greatly among different species and/or cultivars, ranging from 34 (in I. pumila 'Dash Away') to 64 (in I. germanica 'Rare Edition'). The most components $(64,61,60)$ were detected in the three I. germanica cultivars 'Rare Edition', 'Splashacata' and 'Rajah Brooke', respectively, whereas the least components (34, 35 and 35,35 ) were found in two I. pumila cultivars ('Dash Away' and 'Brassie'), as well as two I. germanica cultivars ('Spiced Custard' and 'Wanda Rezac'). In the two I. pallida cultivars ('Dalmatica' and 'Albo Variegata') 36 and 59 components were detected, respectively (Table 2).

In the twenty-seven accessions, twelve cultivars/species had as first dominant scent components terpenes whose relative areas could reach as high as $90.16 \%$ in I. germanica 'Blessed Again', $89.03 \%$ in I. pallida 'Dalmatica' and 74.62\% in I. germanica 'Amsterdam'. 1-caryophyllene accounted for the highest relative content in the accessions of I. germanica 'Casual Elegance', I. germanica 'Lenora Pearls', I. germanica 'Amsterdam' and I. pallida 'Dalmatica', while $\beta$-cedrene in the I. germanica cultivars of 'Blessed Again', 'Indian Chief', 'Summer Olympics' and 'Splashacata'. Besides, I. germanica cultivars of 'Crown Princess', 'Abridged Version', 'Rare Edition' and 'Tulip Festavil' were characterized by the highest level of limonene, thujopsene, tativene and $\alpha$-pinene, respectively. 
Table 1. Floral scent compounds and relative contents emitted from twenty-seven bearded iris accessions.

\begin{tabular}{|c|c|c|c|c|c|c|c|c|c|c|c|c|c|c|c|c|c|c|c|c|c|c|c|c|c|c|c|}
\hline \multirow{2}{*}{ Compound } & \multicolumn{27}{|c|}{ Relative Content for Each Compound (\%) } \\
\hline & 1 & 2 & 3 & 4 & 5 & 6 & 7 & 8 & 9 & 10 & 11 & 12 & 13 & 14 & 15 & 16 & 17 & 18 & 19 & 20 & 21 & 22 & 23 & 24 & 25 & 26 & 27 \\
\hline Methyl cinnamate & 34.16 & & 0.20 & & & 7.71 & & 0.34 & & 13.54 & & 23.59 & 1.67 & 10.48 & & & & & & 0.22 & & & 0.92 & & 2.43 & & 0.23 \\
\hline Isosafrole & 21.27 & & & & & & & 0.15 & & 6.26 & & 14.01 & 1.16 & & & & & & & 0.14 & & & 0.67 & & 0.92 & & \\
\hline Safrole & 5.94 & & & & & & & & & & & & & & & & & & & & & & & & & & \\
\hline Farnesol & 5.31 & & & & 0.15 & & & & & & 0.16 & & 0.21 & & & & & 0.23 & & & 0.96 & & 0.37 & & & 0.16 & \\
\hline$\alpha$-Longipinene & 3.24 & & 0.13 & 0.91 & 7.4 & 0.75 & & 0.15 & & 0.14 & 0.25 & & 1.29 & 0.19 & 0.11 & 1.09 & 1.09 & 2.51 & 2.86 & 0.44 & 0.23 & 1.23 & 0.17 & & & 4.66 & 3.34 \\
\hline Linalyl formate & 3.03 & & & & & & & & & & 0.1 & & & & & & & & & & 0.74 & & & & & & \\
\hline Thujopsene & 2.45 & 1.90 & 18.33 & 1.70 & 0.11 & 0.31 & 0.53 & 0.51 & 10.11 & 2.30 & 1.57 & 0.18 & & 21.92 & 5.95 & 3.82 & 0.22 & 0.31 & 0.37 & 0.25 & 5.36 & 0.24 & 1.18 & 17.58 & 0.85 & & 4.44 \\
\hline Geranyl acetone & 1.59 & & & & & 0.21 & & & & & 0.18 & 0.3 & 0.16 & 0.55 & & 1.91 & & 0.29 & & 0.14 & & & 0.23 & 0.12 & & & 0.65 \\
\hline$\alpha$-Pinene & 1.56 & 3.51 & 5.70 & 0.42 & 0.46 & 0.48 & 0.81 & & 2.19 & 1.69 & 0.83 & 11.1 & 1.15 & & 11.97 & 1.23 & 4.02 & & 1.03 & 0.61 & 10.71 & 3.69 & & & 2.76 & 0.46 & 0.24 \\
\hline Limonene & 1.42 & 16.99 & 2.5 & & 4.89 & & 0.43 & & 1.14 & 0.79 & 0.31 & 11.2 & 0.17 & & 7.63 & 0.21 & 4.49 & & 1.1 & & 2.54 & 3.45 & & & 1.06 & 0.19 & 0.50 \\
\hline Nerolidol & 1.23 & 0.60 & 1.57 & 0.31 & 0.13 & & & 18.15 & & 2.78 & 12.63 & & 11.07 & 0.38 & & 0.61 & 0.61 & 0.19 & 0.10 & 0.60 & 0.62 & & 22.52 & 1.46 & 0.56 & 1.61 & 1.72 \\
\hline Ttetrahydrogeraniol & 1.05 & & 0.35 & 1.38 & & & 0.30 & 0.32 & 0.11 & 1.26 & 0.14 & 0.76 & 0.49 & 0.25 & 0.82 & 19.04 & 0.26 & 3.58 & 0.35 & & 2.72 & 0.28 & 0.69 & 4.12 & & 0.32 & \\
\hline (-)-Verbenone & 0.93 & & 0.19 & & & & & & 0.53 & 0.46 & & 0.24 & & & 1.7 & & & 0.12 & & & & & & & & & 0.96 \\
\hline Menthol & 0.82 & 0.10 & & & & & 0.76 & & & & & & & & & & 0.17 & 0.58 & & & & & & & & & \\
\hline Isolimonene & 0.72 & & & & 1.29 & & & & 0.11 & & & & & & 2.22 & & & & & & & 1.84 & & & 0.44 & & \\
\hline DL-Menthol & 0.67 & & & & & & & & & & & & & & & & & & & & & & 0.17 & & & & \\
\hline Isomenthol & 0.62 & & 0.44 & 0.13 & & & & & & & & & & & & & & & & & & & & & & & \\
\hline Hexadecane & 0.59 & 0.15 & 0.21 & 0.65 & 0.47 & 0.43 & & & 0.14 & 0.18 & & 0.13 & & 0.21 & 0.10 & 0.79 & 0.22 & 0.26 & 0.33 & 0.39 & 0.16 & & & 0.18 & & 0.26 & 0.74 \\
\hline Safranal & 0.55 & & & & & & & & & & & & & & 1.29 & & & & & & & & & & & & \\
\hline $\begin{array}{l}\alpha \text {-Phellandrene } \\
\text { Butyl butyrate }\end{array}$ & $\begin{array}{l}0.47 \\
0.45\end{array}$ & 1.11 & 0.14 & & & & & 0.49 & 0.10 & 0.61 & & & & & & 0.23 & 0.14 & & 0.64 & & & 0.54 & & & & & \\
\hline Citronellyl acetate & 0.44 & & & & & 4.43 & 0.18 & & & & & & & & & & & & 0.10 & & 0.11 & & 0.42 & & 0.42 & & 16.9 \\
\hline 3-Methylheptane & 0.43 & & & & & & & & & & & & & 0.13 & & 0.11 & & 0.12 & & & & & & & & & \\
\hline Farnes & 0.36 & & & & & & & & & & & & & & & 0.23 & & & & & 0.11 & & & & & & \\
\hline Pentadecane & 0.35 & 0.11 & 0.17 & 0.39 & 0.21 & 12.74 & 0.13 & 0.31 & 1.2 & 0.35 & 2.98 & 0.22 & 0.57 & 8.06 & & 0.33 & 0.53 & 0.85 & 6.08 & 7.98 & 0.24 & 0.22 & 0.26 & 0.28 & 0.47 & & 7.79 \\
\hline Butyl isobutyrate & 0.29 & & & 0.10 & & & & & & & & & & & & & & & & & & & & & & & \\
\hline Eugen & 0.27 & & & 3.88 & & & & & & & & & & & & & & & & & 0.27 & & & & & & \\
\hline Trideca & 0.26 & & & 0.39 & & & & & 0.19 & & 0.41 & 0.11 & & 0.74 & & 0.11 & & 0.10 & 0.21 & 3.05 & & & 0.46 & 0.48 & & & 0.31 \\
\hline Cineole & 0.25 & & 0.15 & & & & & & 0.15 & 0.29 & & 0.20 & & & 0.42 & & 0.19 & & & & 0.50 & & & & 0.11 & 0.1 & \\
\hline 1-Octanol & 0.23 & & & & & & & & & 0.14 & & & & & 0.33 & 2.41 & & & & & & & & 1.16 & & & 0.13 \\
\hline Tetrad & 0.22 & 0.12 & 0.10 & 0.2 & & & & & & & & & & 0.13 & & 0.38 & & 0.28 & 0.18 & 0.42 & & & & & & & 0.62 \\
\hline $1-\mathrm{Un}$ & 0.21 & 0.20 & 0.15 & & & & & & & & & 0.17 & & 0.19 & & & & & & & 0.11 & & & 0.16 & & & 0.11 \\
\hline Methyl & 0.21 & & 0.20 & & & 0.42 & & & & & 0.24 & & & 2.5 & & 0.19 & 0.11 & 0.86 & & & & & & & & & \\
\hline Camph & 0.21 & 0.42 & & 0.14 & & & 0.15 & & 0.7 & 1.28 & & 0.50 & & & 2.56 & 0.15 & 1.35 & & & & & 0.83 & & & 0.88 & & 0.26 \\
\hline trans-Dih & 0.21 & & 0.19 & & & & 3.96 & 0.31 & 0.23 & 5.10 & & & & & & & & & & & & & & 0.61 & & & \\
\hline$\beta$-Pinene & 0.20 & 4.39 & 0.20 & & 0.34 & & & 1.47 & 0.15 & 0.24 & & 2.09 & & 0.26 & 1.14 & & 2.26 & 0.44 & 0.20 & & 0.26 & 0.88 & & & 1.86 & & \\
\hline $\mathrm{Ur}_{\mathrm{r}}$ & 0.20 & & & 0.11 & & & & & 0.13 & & & & & 0.24 & 0.1 & 3.93 & & & 0.13 & & & & & 0.27 & & & \\
\hline$\beta$-Terpineol & 0.17 & & & & & & & & & & & 0.17 & & & 0.33 & & & & & 3.78 & & & & & 0.13 & & \\
\hline Cedrol & 0.13 & & & 0.37 & 0.23 & & & & & & & 0.19 & & & & 0.24 & & 0.19 & & 0.10 & 0.13 & & & & & 0.13 & \\
\hline Myrce & 0.13 & 4.38 & 0.75 & & 0.29 & & & 0.31 & 0.30 & 0.34 & & 1.08 & & & 1.38 & & 2.08 & 0.76 & 0.37 & & 0.91 & 0.80 & & & 2.87 & & 0.11 \\
\hline & 0.13 & 0.45 & 0.27 & & & & & & & & & & & & & & 0.85 & & & & 0.26 & 0.22 & & & & & \\
\hline Methyl octanoate & 0.12 & & 0.35 & & & & & & & 0.1 & & 0.14 & & & & 0.59 & & 1.10 & & & & & 0.18 & & & & 0.12 \\
\hline $\begin{array}{l}\text { Lauryl alcohc } \\
\text { Isoeugenol }\end{array}$ & 0.12 & 0.20 & 0.11 & 0.12 & 0.20 & 2.42 & 0.18 & 0.27 & 0.12 & 0.44 & 0.13 & 0.24 & 1.74 & 0.70 & & & 0.22 & 0.50 & & & 0.24 & & 0.62 & 0.53 & 0.59 & 0.36 & 0.32 \\
\hline
\end{tabular}


Table 1. Cont

\begin{tabular}{|c|c|c|c|c|c|c|c|c|c|c|c|c|c|c|c|c|c|c|c|c|c|c|c|c|c|c|c|}
\hline \multirow{2}{*}{ Compound } & \multicolumn{27}{|c|}{ Relative Content for Each Compound (\%) } \\
\hline & 1 & 2 & 3 & 4 & 5 & 6 & 7 & 8 & 9 & 10 & 11 & 12 & 13 & 14 & 15 & 16 & 17 & 18 & 19 & 20 & 21 & 22 & 23 & 24 & 25 & 26 & 27 \\
\hline Geranial & 0.12 & & 0.50 & & & & 0.44 & & & & & & & & & & & & & & & & & & 1.99 & & \\
\hline Terpinyl acetate & 0.12 & 1.67 & 0.10 & & & & & & & 0.18 & & & & & 0.60 & & & & & & & & 4.06 & 0.13 & & & \\
\hline 7,8-Epoxy- $\alpha$-ionone & 0.11 & & & & & & & & & & & & & & & & & & & & & & & 0.11 & & & \\
\hline Nonane & 0.10 & 0.10 & & 0.15 & & & & & & & & & & & & & 0.11 & & & & & & & & & & \\
\hline $\begin{array}{l}\text { Citronellyl } \\
\text { propanoate }\end{array}$ & 0.10 & & & & & 2.58 & 1.25 & & & & & & & & & & & & 0.31 & 2.63 & 0.17 & & 0.43 & & 0.11 & & \\
\hline 3-Carene & 0.10 & 1.49 & 0.98 & & & & 1.32 & & 0.10 & 1.45 & & & & 0.25 & 0.30 & 0.22 & & 0.33 & 0.49 & & & 0.38 & & & 9.23 & & 0.64 \\
\hline 1-Caryophyllene & 0.10 & & 0.10 & 45.44 & 24.64 & 0.38 & & 0.27 & 0.25 & 7.46 & 9.64 & & 17.11 & 0.71 & & & 1.14 & 5.72 & 0.12 & 19.60 & 0.5 & 37.41 & 0.90 & & & 54.38 & 10.71 \\
\hline $\begin{array}{l}\text { Butyl hydroxy } \\
\text { anisole }\end{array}$ & 0.10 & & & & & & & & & & & & & & & 0.12 & & & & & & & & & & & \\
\hline Methyl myristate & & 22.86 & & & & & 2.55 & 2.67 & 7.87 & & 2.10 & 9.72 & 5.40 & 3.30 & & 1.93 & 20.25 & 2.29 & & 0.83 & 7.76 & & 5.562 & 2.85 & & & 0.70 \\
\hline Anisic aldehyde & & 5.30 & & & 0.24 & & & & & & & & & & & & & & & & & & & 1.44 & & & \\
\hline Isoprene & & 3.30 & & & 0.13 & & & & & & & & & & & & & & & & & 0.12 & & & & & \\
\hline Linalool & & 3.14 & 11.88 & 10.00 & & 4.98 & 10.25 & 21.79 & 32.42 & 13.41 & 12.22 & & 18.17 & & 33.84 & 0.25 & 0.33 & & 18.26 & 16.29 & 17.90 & 10.75 & 18.683 & 34.61 & 4.37 & & 0.12 \\
\hline 4-Carene & & 2.73 & & & 0.35 & & & & 0.26 & & & 0.64 & & & 0.63 & & 2.45 & & & & & 0.38 & & & & & \\
\hline Linalyl acetate & & 1.92 & 0.19 & & & 4.20 & 4.78 & & 6.31 & 1.5 & 1.74 & & 0.69 & & 9.42 & & & & 11.16 & 8.66 & 8.79 & 7.33 & & 21.99 & & & 0.10 \\
\hline $\begin{array}{c}\text { Geranyl } \\
\text { phenylacetate }\end{array}$ & & 1.89 & & & & & & & & & & & & & 0.83 & & 0.24 & & & & & & & & & & \\
\hline $\begin{array}{c}\text { Ethyl } \\
\text { chrysanthemumate }\end{array}$ & & 1.67 & & & & & & & & & & & & & & & & & & & & & & 1.98 & & & \\
\hline Alloaromadendrene & & 1.54 & & 1.19 & 2.8 & 1.95 & & 0.87 & 2.66 & 1.53 & 5.58 & & 0.39 & 11.75 & 1.20 & 5.21 & 0.13 & 0.38 & 5.50 & & 5.64 & 5.47 & & & & 0.65 & 0.16 \\
\hline Methyl laurinate & & 1.41 & & & & & & & 0.30 & & & 0.69 & 0.33 & & & 1.67 & 3.11 & 9.54 & & & 0.14 & & & 0.30 & & & 0.69 \\
\hline Sativene & & 1.33 & & 1.08 & 0.19 & 2.02 & & 0.20 & 0.76 & & 0.53 & & 0.13 & 0.46 & 0.69 & 7.80 & & 1.63 & & 0.58 & 1.64 & & & & & 0.41 & \\
\hline $\begin{array}{c}\text { Methyl } \\
\text { hexadecanoate }\end{array}$ & & 1.03 & & & & & 0.91 & 0.60 & 6.95 & & 1.57 & 5.51 & 3.78 & 1.30 & & 6.01 & 23.18 & 12.03 & & 0.24 & 3.44 & & 6.77 & 0.85 & & & 5.64 \\
\hline Methyl undecanoate & & 0.84 & & & & & & 0.14 & & & & & 0.10 & 1.21 & & 0.22 & 0.22 & 0.19 & & & 0.13 & & 0.25 & 0.10 & & & 1.38 \\
\hline$\beta$-Cyclocitral & & 0.58 & & & & & & & & & & & & & & & & & & & & & & & 0.69 & & \\
\hline$\alpha$-Guaiene & & 0.57 & & 0.30 & 1.22 & 0.33 & & 0.33 & & & & & & 0.36 & 0.11 & 0.18 & & 0.19 & & & 0.32 & & & & & 0.28 & 0.13 \\
\hline Ethylbenzene & & 0.53 & 0.50 & & & & 0.29 & & & & & & & & & & 0.86 & & & 0.19 & & & & & & & \\
\hline$\alpha$-Terpinene & & 0.46 & 0.16 & & 0.23 & & & & & & & 0.17 & & & & & 0.21 & & & & & 0.22 & & & 0.10 & & \\
\hline trans-Carveol & & 0.32 & & & & & & & & & & & & & & & & & & & & & & & & & \\
\hline$\alpha$-Bulnesene & & 0.30 & & & 0.96 & 0.8 & & 1.55 & & 0.63 & 2.49 & & & 0.31 & & 2.57 & & 0.45 & 0.10 & & & 0.52 & & & & 1.32 & \\
\hline (-)-4-Terpineol & & 0.24 & 0.26 & & & & 0.13 & & 0.18 & 0.13 & & 0.60 & 0.11 & & 0.23 & & 0.59 & & & & & 0.41 & & & & 0.15 & 0.12 \\
\hline Calarene & & 0.17 & & 0.35 & 2.01 & & & & & & & & 1.30 & 0.22 & & & 0.76 & & 3.63 & & 0.31 & 0.34 & & & & & \\
\hline 1-Decanol & & 0.17 & & & & & & & & & & & & 0.16 & 0.79 & & & & & & 0.12 & & & 0.53 & & & \\
\hline Valencene & & 0.17 & & & 0.17 & 0.12 & & 0.46 & 0.34 & & 0.64 & & & 1.2 & 0.20 & 0.38 & & 0.21 & 3.76 & & 0.70 & & & & & & 0.15 \\
\hline 1-Methoxy-4-propylbenz & zene & 0.16 & & & & & & & & & & & & & & & & & & & & & & & & & \\
\hline Isocyclocitral & & 0.11 & & & & & & & & & & & & & & & & & & & & 0.10 & & & & & \\
\hline $\begin{array}{c}\text { Methyl } \\
\text { 10-undecenoate }\end{array}$ & & 0.11 & & & & & & & 0.16 & & 0.24 & 0.50 & & & & 0.57 & 0.30 & 0.47 & & & 0.24 & & & 0.13 & & & 0.24 \\
\hline Geraniol & & & 22.64 & & & & 5.7 & & & & & & & & 0.25 & 0.63 & 1.91 & & & & & & 2.11 & & 2.87 & & 1.99 \\
\hline 2-Pentadecanone & & & 5.91 & & & & & & & & & & & & & & & & & & & & & & & & \\
\hline$\alpha$-Caryophyllene & & & 4.82 & 0.41 & 0.67 & & 0.12 & 1.03 & 0.14 & 0.28 & & & & 16.70 & & & 0.18 & 1.63 & 0.11 & & & 3.95 & & 0.34 & & 0.71 & \\
\hline Nerol & & & 3.43 & & & & 12.40 & & & 0.15 & & & & & & 1.85 & 0.18 & & 1.33 & 0.54 & & 3.90 & 0.13 & & 23.25 & 0.55 & 0.21 \\
\hline Geranyl butyrate & & & 1.03 & & & & 0.13 & & & & & & & & & & 1.42 & & & & & & & & & & \\
\hline Neryl acetate & & & 0.87 & & & & 0.28 & & & & & & & & & & 1.92 & & & & & & 1.39 & & 2.40 & & \\
\hline 2-Dodecanone & & & 0.76 & & & & & & & & & & & & & & & & & & & & & & & & \\
\hline Methyl caprate & & & 0.71 & & & & & & & & & & 0.4 & & & 6.60 & & 14.08 & & 0.13 & & & & 0.61 & & & 2.87 \\
\hline
\end{tabular}


Table 1. Cont

\begin{tabular}{|c|c|c|c|c|c|c|c|c|c|c|c|c|c|c|c|c|c|c|c|c|c|c|c|c|c|c|c|}
\hline \multirow{2}{*}{ Compound } & \multicolumn{27}{|c|}{ Relative Content for Each Compound (\%) } \\
\hline & 1 & 2 & 3 & 4 & 5 & 6 & 7 & 8 & 9 & 10 & 11 & 12 & 13 & 14 & 15 & 16 & 17 & 18 & 19 & 20 & 21 & 22 & 23 & 24 & 25 & 26 & 27 \\
\hline Isopulegol & & & 0.62 & & & 0.10 & 0.12 & & 0.37 & 0.66 & 0.13 & 0.70 & & & 0.80 & & 0.16 & & 0.33 & & 0.69 & 1.07 & 0.33 & & 0.22 & & \\
\hline Phenylacetaldehyde & & & 0.58 & & & & & 0.17 & & & & & & & & & & & & & & & & & & & \\
\hline Methyl toluene & & & 0.47 & 0.15 & & & & & & & & & & & & & & & 0.39 & & & & & & & & \\
\hline$\alpha$-Terpineol & & & 0.40 & & & & & & 0.46 & 0.83 & 0.62 & 0.63 & & & 1.09 & & 0.95 & & 0.36 & & 0.64 & 0.81 & 1.19 & & 0.36 & & \\
\hline Citronellyl formate & & & 0.37 & & & & 0.12 & & & & & & & & & 0.16 & & & & & & & & & & 0.17 & \\
\hline Tridecanal & & & 0.36 & 0.11 & & & & & & & & & & & & & & 0.36 & & & & & & & & & \\
\hline$\alpha$-Santalol & & & 0.35 & 0.47 & 0.70 & 0.12 & & & & 0.13 & & & & & & & & 0.25 & & & & & & & & 0.10 & 0.10 \\
\hline Citronellol & & & 0.27 & & & & 33.64 & & & & & & & & & 0.55 & 0.26 & & & & & & 2.14 & & 25.58 & & \\
\hline Diethyl carbonate & & & 0.26 & & & & & & & & & & & & & & & & & & & & & & & & \\
\hline cis-Citral & & & 0.20 & & & & 0.12 & & & & & & & & & & & & & & & & & & 0.87 & & \\
\hline Tricyclene & & 1.79 & 0.18 & & & & 0.16 & & & & & & & & 0.35 & 0.81 & & & 0.15 & & 3.37 & & & & 0.16 & & \\
\hline Linalool propionate & & & 0.18 & & & & 0.27 & & & & & & & & & & 0.13 & & 0.11 & 0.10 & 5.10 & & 2.27 & & 0.16 & & \\
\hline Undecanal & & & 0.14 & & & & & & & & & 0.18 & & 0.10 & & & & & & & & & & & & & 0.13 \\
\hline Cumin alcohol & & & 0.14 & & & & & & & & & & & & & & & & & & & & & & & & \\
\hline Tridecanol & & & 0.13 & & & 9.65 & & & & 0.17 & & & & 0.25 & & 0.14 & & & 0.19 & 3.57 & 0.12 & & & & & & 0.11 \\
\hline Aromadendrene & & & 0.12 & 0.26 & 0.84 & & & 1.22 & & & & & & 1.40 & & 0.57 & & & & 0.34 & 0.12 & & & & & & \\
\hline Methyl benzoate & & & 0.11 & & 0.11 & & & & & & & & & & & & & & & & 0.65 & & & & & 0.14 & 0.15 \\
\hline Tetradecanal & & & 0.10 & 0.12 & 0.19 & 0.53 & 0.28 & 0.32 & 0.65 & 1.86 & 0.27 & & 0.14 & 1.81 & & 0.76 & 1.94 & 0.86 & & & & & 0.51 & & & 0.18 & \\
\hline$\alpha$-Bisabolene & & & & 7.17 & 1.77 & 0.24 & & 0.21 & 0.15 & & 1.30 & & 1.03 & & & & & 0.40 & 0.90 & 0.10 & & 0.16 & & & & 4.12 & \\
\hline$\alpha$-Copaene & & & & 7.12 & & & & & & & 0.16 & & 0.27 & & & & 6.80 & & 2.26 & 0.19 & & & & & & & \\
\hline Ketone & & & & 2.34 & & & & & & & & & & & & & & & & & & & & & & & 0.17 \\
\hline$\beta$-Cedrene & & & & 1.53 & 29.36 & 5.56 & 0.13 & 16.94 & & 9.89 & 19.29 & & 13.89 & & & 3.41 & & 12.92 & 11.07 & 1.63 & & 8.66 & & & & 7.10 & 3.20 \\
\hline$\alpha$-Cubebene & & & & 1.05 & & & & & & & & & & 0.81 & & & 1.15 & & 0.65 & & & & & & & & \\
\hline$\alpha$-Cedrene & & & & 0.90 & 2.16 & 0.21 & & 2.51 & 1.37 & 1.35 & 2.69 & & 1.72 & & & & 0.17 & 0.34 & 1.12 & 0.26 & & 0.79 & & & & 1.53 & 0.24 \\
\hline$\alpha$-Bisabolol & & & & 0.79 & 0.39 & & & 0.18 & & 0.12 & 0.27 & & 0.17 & & & & & & 0.12 & 1.54 & & & & & & 0.10 & \\
\hline 8-Cadinene & & & & 0.68 & & & & & & & & & & 0.10 & & 0.19 & & & 0.29 & & & & & & & 10.64 & \\
\hline Patchouli alcohol & & & & 0.59 & & & & & & & & & & & & & & & & & & & & & & 0.11 & \\
\hline $\begin{array}{l}\text { Propylbenzene } \\
\alpha \text {-Asarone }\end{array}$ & & & & $\begin{array}{l}0.45 \\
0.42\end{array}$ & & & & 0.17 & & & & & 0.44 & & & & & & & & & & & & & & \\
\hline Heptadecane & & & & 0.36 & 0.41 & 7.68 & & & 0.18 & 0.19 & 0.11 & 0.16 & 0.13 & 1.05 & 0.22 & & 0.28 & 0.10 & 0.42 & 0.38 & & & & & & 0.17 & 0.50 \\
\hline Eugenol acetate & & & & 0.35 & & & & & & & & & & & & & & & & & 0.19 & & & & & & \\
\hline Piperonal & & & & 0.35 & & & & & & 0.1 & & & & & & & & 0.33 & & 0.10 & & & 0.10 & & & & 0.16 \\
\hline Viridiflorene & & & & 0.28 & & & & 3.11 & & & 0.32 & & 1.84 & 0.12 & & 0.21 & & & 0.18 & & & & 11.76 & & & & \\
\hline 2-Ethylhexanol & & & & 0.27 & & & & & & & & & & & & & & & & & & & & & & & \\
\hline$\beta$-Asaron & & & & 0.18 & & & & & & & & & & & & & & & & & & & & & & & \\
\hline Globulol & & & & 0.17 & & & & 8.07 & & & 0.12 & & & & & & 0.60 & & & & & & & & & & \\
\hline$\alpha$-Longifolene & & & & 0.17 & 4.45 & & & & 0.15 & 1.47 & 2.54 & & 0.17 & 0.17 & & 0.39 & & & 0.22 & 0.23 & 1.99 & 1.18 & & & & 2.28 & \\
\hline Caryophyllene oxide & & & & 0.16 & 0.10 & & & & & 0.12 & & & & 0.23 & & & 0.17 & & & & & & & 0.30 & & 0.12 & \\
\hline$\beta$-Santalol & & & & 0.16 & 0.37 & & & & & 0.13 & 0.17 & & 0.26 & & & & & 0.17 & & & & 0.18 & & & & 0.34 & \\
\hline$\gamma$-Gurjunene & & & & 0.14 & 0.10 & 1.11 & & 3.89 & & & 0.84 & & & 0.24 & 0.11 & 0.43 & & 0.10 & & & 0.13 & & & & & & \\
\hline 1-Isoproyl-3-tert-butylbe & nzene & & & 0.13 & & & & & & & & & & & & & 0.25 & & & & & & & & & & \\
\hline (-)-Aristolene & & & & 0.11 & & & & & & & & & & & & & & & 0.23 & 1.26 & & & & & & & \\
\hline 2-tert-Butyl-4-hydroxyan & isole & & & 0.10 & & & & & & & & & & & & & & & & & & & & & & & \\
\hline 2-Isopropyl-5-ketohexan & & & & 0.10 & & & & & & & & & & & & & & & & & & & & & & & \\
\hline$\beta$-Eudesmene & & & & & 2.67 & & & 0.15 & & & & & & & & & & & & 0.34 & 0.16 & & & & & 0.17 & 0.11 \\
\hline$\rho$-Diethylbenzene & & & & & 0.60 & & & 0.36 & & & 0.64 & & 0.26 & & & & & & & & & & & & & & \\
\hline Benzyl formate & & & & & 0.36 & & & & & & & & & & & & & & & & & & & & & & \\
\hline Phenylethyl alcohol & & & & & 0.32 & & & & & & & 1.43 & & & & & & & & & & 0.28 & & 0.76 & & & \\
\hline
\end{tabular}


Table 1. Cont

\begin{tabular}{|c|c|c|c|c|c|c|c|c|c|c|c|c|c|c|c|c|c|c|c|c|c|c|c|c|c|c|c|}
\hline \multirow{2}{*}{ Compound } & \multicolumn{27}{|c|}{ Relative Content for Each Compound (\%) } \\
\hline & 1 & 2 & 3 & 4 & 5 & 6 & 7 & 8 & 9 & 10 & 11 & 12 & 13 & 14 & 15 & 16 & 17 & 18 & 19 & 20 & 21 & 22 & 23 & 24 & 25 & 26 & 27 \\
\hline$\gamma$-Terpinene & & & & & 0.30 & & & & & & 1.71 & & & & 0.26 & & 0.15 & & & & & 0.17 & & & & & \\
\hline Phenylacetic acid & & & & & 0.22 & & & & & & & & & & & & & & & & & & & & & & \\
\hline Dolcymene & & & & & 0.17 & 0.26 & & 0.18 & & & 3.88 & & 0.11 & & & & & & 1.71 & 0.34 & & 0.26 & & & & & \\
\hline Terpinolene & & & & & 0.15 & 0.11 & & & & & & & & & & & 1.12 & & & & & 0.91 & & & & & \\
\hline 2-Phenyl-1-propanal & & & & & 0.11 & & & & & & 0.11 & & & & & 0.24 & & & & & & & & & & 0.23 & \\
\hline Isolongifolene & & & & & 0.10 & & & & 0.16 & & & & & & & & & & & & & & & & & & \\
\hline$\alpha$-Thujone & & & & & & 13.41 & & & & & & & & & & 0.43 & & 0.11 & 1.47 & 2.58 & & & & & & & 6.1 \\
\hline 2,2,4-Trimethylpentane & & & & & & 2.71 & & & & & & & & & & & & & & & & & & & & & \\
\hline Myristyl alcohol & & & & & & 2.33 & 0.22 & 2.02 & & 0.32 & & & & 0.18 & & & 0.11 & & 0.54 & 3.1 & & & & & & & 0.14 \\
\hline Cryptone & & & & & & 2.26 & & & & & & & & & & & & & & 2.64 & & & & & & & 0.36 \\
\hline 2,5-Dimethylhexane & & & & & & 0.17 & & & & & & 0.13 & & 0.49 & 0.32 & 6.47 & 0.18 & & 0.22 & & & & & 0.21 & & & \\
\hline Dihydrocarvone & & & & & & 0.16 & & & & & & & & & & & & & 0.12 & & & & & & & & 0.13 \\
\hline Methyl linoleate & & & & & & 0.16 & & & & & & & & 1.21 & & 0.16 & & 0.32 & & & & & & & & & \\
\hline Methyl stearate & & & & & & 0.13 & & & & & & & & 0.74 & & & 0.3 & 0.35 & & & & & & & & & \\
\hline Allyl hexanoate & & & & & & 0.11 & & & & & & & & & & 0.46 & & 0.18 & & & & & & & & & \\
\hline 2,4-Dimethylpentane & & & & & & 0.10 & & & & & 0.10 & & & & & & & & & & & & & & & & \\
\hline Cyclohexaneethanol & & & & & & & 5.45 & & & & & & & & & & & & 1.68 & & & & & & & & \\
\hline Methyl nonanoate & & & & & & & 2.00 & & & & & & & & & 0.34 & & 1.39 & & & 0.11 & & & & & & 0.19 \\
\hline Geranic acid & & & & & & & 1.06 & & & & & & & & & & & & & & & & & & 0.37 & & \\
\hline Menthol acetic ester & & & & & & & 0.85 & & & & & & & & & & & & & & & & 0.34 & & 0.24 & & \\
\hline Citronellyl butyrate & & & & & & & 0.72 & & & & & & & & & & & & & & 0.15 & & & & & & 0.26 \\
\hline Crotonaldehyde & & & & & & & 0.46 & & & & & & & & & & & & & & & & & & & & \\
\hline Carvomenthene & & & & & & & 0.19 & & & & & & & & & & & & & & & & & & & & 0.10 \\
\hline Methyl salicylate & & & & & & & 0.18 & & & & & & & & & & & & & & & & & & & & \\
\hline Benzyl benzoate & & & & & & & 0.12 & & & & & & & 0.14 & & & & & & & & & & & & & \\
\hline Benzoic aldehyde & & & & & & & 0.10 & & & & & & & & & & & & & & & & & & & & \\
\hline $\begin{array}{l}m \text {-Isopropyl } \\
\text { ethylbenzene }\end{array}$ & & & & & & & & 0.97 & & & & & & & & & & & 0.34 & & & & & & & & \\
\hline Cinnamaldehyde & & & & & & & & 0.23 & & 9.00 & & 0.81 & & & & & & & & & & & & & & & \\
\hline$m$-Tolualdehyde & & & & & & & & 0.22 & & & & & 0.48 & & & & & & & & & & & & & & 4.36 \\
\hline Amyl caprylate & & & & & & & & 0.16 & & 0.11 & & & & & & & & & & & & & & & & & \\
\hline Longicyclene & & & & & & & & 0.16 & & & & & 0.14 & & & & & & 0.11 & & 1.38 & 0.23 & & & & & \\
\hline Phenetole & & & & & & & & 0.12 & & & & & & & & & & & & & & & & & & & \\
\hline Fenchole & & & & & & & & & 13.59 & & & & & & & & & & & & & & & & 0.86 & & \\
\hline Pantolactone & & & & & & & & & 1.79 & & & & & & & & & & 1.35 & & 2.01 & & 0.75 & & & & \\
\hline Methyl capronate & & & & & & & & & 0.14 & & & & & & & & & & & & & & & & & & \\
\hline Eicosane & & & & & & & & & 0.11 & & & & & & 0.16 & & & 0.11 & & & & & & & & 0.17 & 0.91 \\
\hline$m$-Diethylbenzene & & & & & & & & & & 0.42 & & & & 0.99 & & & & & 0.72 & & & & & & & & \\
\hline 1-Nonanol & & & & & & & & & & 0.13 & & & & & & 0.41 & & & & & & & & & & & \\
\hline$o$-Xylene & & & & & & & & & & 0.13 & & & & & & & & & & & & & & & & & \\
\hline Thymol & & & & & & & & & & 0.12 & & & & & & & & & & & & & & & & & \\
\hline Butyl caprylate & & & & & & & & & & 0.10 & 0.12 & & & & & & & & & & & & & & & & \\
\hline Geranyl tiglate & & & & & & & & & & & 2.31 & & & & & & & & 1.49 & & & & & & & & \\
\hline$\gamma$-Nonanolactone & & & & & & & & & & & 0.99 & & 0.21 & 0.15 & & 0.48 & 0.23 & 0.12 & & 1.58 & & & & & & & \\
\hline$\gamma$-Decalactone & & & & & & & & & & & 0.90 & & & & & 0.26 & & & & & & & & & & & \\
\hline 2,3,4-Trimethylpentane & & & & & & & & & & & 0.43 & & & & & 0.60 & & & & & & & 0.69 & 0.16 & & & \\
\hline $\begin{array}{l}\text { Tetrahydrofurfuryl } \\
\text { acetate }\end{array}$ & & & & & & & & & & & 0.31 & & & & 0.58 & & & & & 1.42 & & & & & & & \\
\hline
\end{tabular}


Table 1. Cont.

\begin{tabular}{|c|c|c|c|c|c|c|c|c|c|c|c|c|c|c|c|c|c|c|c|c|c|c|c|c|c|c|}
\hline \multirow{2}{*}{ Compound } & \multicolumn{26}{|c|}{ Relative Content for Each Compound (\%) } \\
\hline & 1 & 3 & 4 & 5 & 6 & 7 & 8 & 9 & 10 & 11 & 12 & 13 & 14 & 15 & 16 & 17 & 18 & 19 & 20 & 21 & 22 & 23 & 24 & 25 & 26 & 27 \\
\hline Nootkanone & & & & & & & & & & 0.13 & & & & & & & & & & 0.15 & & & & & & \\
\hline$\alpha$-Cyclociral & & & & & & & & & & 0.11 & & & & 0.10 & & & & & & & & & & & & \\
\hline $\begin{array}{l}\text { Hydroquinone } \\
\text { Dimethyl }\end{array}$ & & & & & & & & & & & 4.93 & & & & & & 8.82 & & & 2.2 & & & & & & \\
\hline Mesitylene & & & & & & & & & & & & 0.45 & & & & & & & & & & & & & & \\
\hline Linalyl isovalerate & & & & & & & & & & & & 0.36 & & & & & & & & & & & & & & \\
\hline Methyl acetate & & & & & & & & & & & & 0.21 & & & & & & & & & & & & & & \\
\hline Methyl $m$-tolylketone & & & & & & & & & & & & 0.20 & & & & & & 0.84 & & & & & & & & \\
\hline 2,2,5-Trimethylhexane & & & & & & & & & & & & & 0.35 & & & & & & 0.14 & & & & & & & \\
\hline Dodecane & & & & & & & & & & & & & 0.13 & & 0.52 & & & & & & & & 0.19 & & & \\
\hline Octyl formate & & & & & & & & & & & & & & 0.33 & 0.27 & & 0.20 & & & 0.36 & & & 0.23 & & & \\
\hline Cyclohexane & & & & & & & & & & & & & & & 0.79 & & 0.22 & & & & & & & & & \\
\hline 2,6-Di-tert-butyl-p-cresol & & & & & & & & & & & & & & & 0.58 & 0.11 & & & & 0.13 & & & & & & 0.10 \\
\hline$\gamma$-Valerolactone & & & & & & & & & & & & & & & 0.42 & & & & & & & & & & & \\
\hline 6-Decalactone & & & & & & & & & & & & & & & 0.17 & & & & & & & & 0.11 & & & \\
\hline Dihydro- $\alpha$-ionone & & & & & & & & & & & & & & & 0.13 & & & & & & & & & & & \\
\hline trans-2-Nonenal & & & & & & & & & & & & & & & & 0.10 & & & & & & & & & & \\
\hline trans-2-Pinanol & & & & & & & & & & & & & & & & 0.38 & & & & & & & & & & 0.27 \\
\hline 2,2,4,4-Tetramethyl-3-pent & tanone & & & & & & & & & & & & & & & 0.20 & & & & & & & 0.27 & & & \\
\hline $\begin{array}{l}\text { 2-Methylpropanoic } \\
\text { acetyl ester }\end{array}$ & & & & & & & & & & & & & & & & & 2.10 & 0.29 & 0.32 & & & & & & & \\
\hline 2,3-Dimethylcyclohexanol & & & & & & & & & & & & & & & & & 0.39 & & & & & & & & & \\
\hline Decanal & & & & & & & & & & & & & & & & & 0.25 & & & & 0.13 & & & & & \\
\hline$\beta$-Pinone & & & & & & & & & & & & & & & & & 0.15 & & & & & & & & & \\
\hline 3-Methylpentane & & & & & & & & & & & & & & & & & 0.11 & & & & & & 1.05 & & & \\
\hline$\beta$-Thujone & & & & & & & & & & & & & & & & & & 2.58 & & & & & & & & \\
\hline Isohexane & & & & & & & & & & & & & & & & & & 1.12 & & & & & & & & \\
\hline Neohexane & & & & & & & & & & & & & & & & & & 0.82 & & & & & & & & \\
\hline$p$-Methylbenzaldehyde & & & & & & & & & & & & & & & & & & 0.24 & & & & & & & 0.51 & \\
\hline Pentanol & & & & & & & & & & & & & & & & & & 0.18 & & & & & & & & \\
\hline Citronellene & & & & & & & & & & & & & & & & & & & 2.71 & & & & & & & \\
\hline Pentadecanol & & & & & & & & & & & & & & & & & & & 0.19 & & & & & & & \\
\hline 2-Ethyl-1-butanol & & & & & & & & & & & & & & & & & & & 0.19 & & & & & & & \\
\hline Cedryl acetate & & & & & & & & & & & & & & & & & & & & 0.21 & & & & & & \\
\hline Myristic acid & & & & & & & & & & & & & & & & & & & & 0.10 & & & & & & \\
\hline Isoamyl butyrate & & & & & & & & & & & & & & & & & & & & & & & 0.13 & & & \\
\hline Perillyl aldehyde & & & & & & & & & & & & & & & & & & & & & & & & 0.12 & & \\
\hline sogeraniol & & & & & & & & & & & & & & & & & & & & & & & & 0.10 & & \\
\hline Methyl anthranilate & & & & & & & & & & & & & & & & & & & & & & & & & & 7.76 \\
\hline $\begin{array}{l}\text { Piperitone } \\
\text { P }\end{array}$ & & & & & & & & & & & & & & & & & & & & & & & & & & 3.01 \\
\hline 3,5-Dimethyl-2-cyclohexer & n-1-one & & & & & & & & & & & & & & & & & & & & & & & & & 2.64 \\
\hline
\end{tabular}


Table 2. Relative contents (\%) and number of components for 10 floral scent compound categories emitted from twenty-seven bearded iris accessions.

\begin{tabular}{|c|c|c|c|c|c|c|c|c|c|c|c|}
\hline Code & Terpene & Alkane & Aromatic & Ester & Alcohol & Aldehyde & Ketone & Ether & Phenol & Acid & Total \\
\hline 1 & $10.71(12)$ & $2.15(7)$ & & $39.29(10)$ & $11.03(13)$ & $0.67(2)$ & $2.63(3)$ & 27.31 & $0.39(2)$ & & $94.18(52)$ \\
\hline 2 & 47 (19) & $0.47(4)$ & $0.53(1)$ & $33.4(9)$ & $4.97(8)$ & $5.99(3)$ & & $0.16(1)$ & & & $92.51(45)$ \\
\hline 3 & 34.39 (14) & $0.49(3)$ & $0.97(2)$ & 4.58 (12) & 43.08 (17) & $1.88(6)$ & $6.86(3)$ & & & & $92.24(57)$ \\
\hline 4 & $71.50(22)$ & $2.23(7)$ & $0.73(3)$ & $0.46(2)$ & $14.76(12)$ & $0.67(4)$ & $2.34(1)$ & $0.70(3)$ & $4.03(2)$ & & $97.42(56)$ \\
\hline 5 & $90.16(29)$ & 1.09 (3) & $0.77(2)$ & $0.47(2)$ & $2.49(8)$ & $0.54(3)$ & & & & $0.22(1)$ & $95.74(48)$ \\
\hline 6 & $14.38(14)$ & $23.83(6)$ & $0.26(1)$ & $19.73(8)$ & $19.61(6)$ & $0.53(1)$ & $16.04(4)$ & & & & $94.38(40)$ \\
\hline 7 & $3.85(9)$ & $0.13(1)$ & $0.29(1)$ & $14.35(14)$ & 73.11 (12) & $1.41(5)$ & & & & $1.06(1)$ & $94.21(43)$ \\
\hline 8 & $35.83(20)$ & $0.31(1)$ & $1.68(4)$ & $3.91(5)$ & $51.11(8)$ & $0.94(4)$ & & $0.26(2)$ & & & $94.03(44)$ \\
\hline 9 & 21.14 (19) & $1.95(6)$ & & $23.52(7)$ & $47.62(9)$ & $0.65(1)$ & $0.53(1)$ & & & & $95.42(43)$ \\
\hline 10 & 31.58 (17) & $0.73(3)$ & $0.54(2)$ & $15.52(6)$ & 26.19 (17) & $10.96(3)$ & $0.46(1)$ & $6.26(1)$ & $0.12(1)$ & & $92.37(51)$ \\
\hline 11 & 50.69 (17) & $4.03(5)$ & $4.52(2)$ & 10.62 (11) & $26.59(10)$ & 0.49 (3) & $0.30(2)$ & & & & $97.25(50)$ \\
\hline 12 & $26.96(8)$ & $0.75(5)$ & & $40.14(6)$ & $5.10(10)$ & $0.99(2)$ & $0.54(2)$ & $18.94(2)$ & & & 93.41 (35) \\
\hline 13 & $40.61(14)$ & $0.70(2)$ & $1.26(4)$ & 13.16(10) & $32.20(8)$ & $0.62(2)$ & $0.36(2)$ & $1.16(1)$ & & & $90.08(43)$ \\
\hline 14 & 57.41 (19) & $11.52(10)$ & 0.99 (1) & $21.03(9)$ & $2.12(7)$ & $1.90(2)$ & $0.55(1)$ & & & & $95.53(49)$ \\
\hline 15 & 36.81 (17) & $0.90(5)$ & & $11.76(5)$ & $38.87(10)$ & $1.39(2)$ & $1.70(1)$ & & & & $91.44(40)$ \\
\hline 16 & $28.85(18)$ & $14.03(10)$ & & $20.96(19)$ & $26.14(10)$ & $1.00(2)$ & $2.47(3)$ & $0.12(1)$ & $0.58(1)$ & & $94.16(64)$ \\
\hline 17 & $30.90(21)$ & $1.31(5)$ & $1.11(2)$ & $51.41(12)$ & $7.05(16)$ & $2.03(2)$ & $0.20(1)$ & & $0.11(1)$ & & $94.13(60)$ \\
\hline 18 & $28.32(16)$ & $2.15(9)$ & & $45.20(15)$ & $6.06(9)$ & $1.79(4)$ & $0.67(4)$ & $8.82(1)$ & & & $93.02(58)$ \\
\hline 19 & $37.45(25)$ & $9.52(9)$ & $3.16(4)$ & $14.81(7)$ & 23.45 & $0.24(1)$ & $5.00(4)$ & & & & $93.63(61)$ \\
\hline 20 & $28.54(14)$ & $12.36(6)$ & $0.53(2)$ & $17.13(10)$ & $29.90(10)$ & $0.10(1)$ & $5.37(3)$ & $0.14(1)$ & & & $93.06(47)$ \\
\hline 21 & 36.84 (19) & $0.40(2)$ & & 30.41 (18) & $24.75(12)$ & & $0.15(1)$ & $2.20(1)$ & $0.40(2)$ & $0.10(1)$ & $95.25(56)$ \\
\hline 22 & $74.62(26)$ & $0.22(1)$ & $0.26(1)$ & & $17.69(8)$ & $0.22(2)$ & & & & & $93.02(38)$ \\
\hline 23 & $14.00(4)$ & $1.42(3)$ & & 30.68 (13) & 48.95 (11) & $0.62(2)$ & $0.23(1)$ & $0.67(1)$ & & & $96.57(35)$ \\
\hline 24 & $18.22(3)$ & $2.81(8)$ & & $29.43(12)$ & $43.93(9)$ & $1.44(1)$ & $0.50(1)$ & & & & $96.33(34)$ \\
\hline 25 & $24.22(10)$ & $0.47(1)$ & & $5.76(6)$ & 59.00 (12) & $3.68(4)$ & & $0.92(1)$ & & $0.37(1)$ & $94.42(35)$ \\
\hline 26 & $89.03(16)$ & $0.60(3)$ & & $0.14(1)$ & $4.04(12)$ & $1.09(4)$ & & & & & $94.89(36)$ \\
\hline 27 & $24.32(15)$ & $10.87(6)$ & & 37.24 (14) & $5.34(12)$ & $4.66(3)$ & $14.02(8)$ & & $0.10(1)$ & & $96.54(59)$ \\
\hline
\end{tabular}


Five cultivars/species had as first dominant scent components esters whose relative areas could reach as high as $51.41 \%$ in I. germanica 'Rajah Brooke', $45.20 \%$ in I. germanica 'Superstition' and $39.29 \%$ in I. germanica wild species. Methyl cinnamate accounted for the highest relative content in the accessions of I. germanica wild species and I. germanica 'Spiced Custard' while methyl hexadecanoate, methyl caprate and citronellyl acetate in I. germanica 'Rajah Brooke', 'Superstition' and I. pallida 'Albo Variegata', respectively. Nine accessions, including seven I. germanica cultivars and two I. pumila cultivars, had as the first dominant scent components alcohols, of which the top three highest levels were detected in the I. germanica cultivars 'Forever Blue' (73.11\%) and 'Wanda Rezac'(48.95\%), and I. pumila 'Brassie' $(59.00 \%)$, mainly including the compounds linalool, citronellol, geraniol and nerolidol. I. germanica 'Hold and Behold' had the highest relative content of alkanes $(23.83 \%)$, six of which were detected with the highest level corresponding to pentadecane (12.74\%).

Differentiations in scent profiles among species or cultivars have been demonstrated in several ornamental plants. It is noteworthy that overall our samples exhibited more volatile complexity than Chrysanthemum (193 compounds across 39 accessions) [24], tree peony (146 compounds across 30 accessions) [6], Narcissus (84 compounds across nine accessions) [25], Hosta (70 compounds across 46 accessions) [13], Silene (60 compounds across 10 species) [26], Phlox (59 compounds across 22 accessions) [27] and so forth. In our study, $\alpha$-longipinene, thujopsene, $\alpha$-pinene, limonene, nerolidol, tetrahydrogeraniol, hexadecane, pentadecane, lauryl alcohol, l-caryophyllene, linalool, alloaromadendrene were detected in most of the accessions. Moreover, we found that the scent profile varied significantly among and within the three investigated species of bearded iris. For instance, although the two I. pumila cultivars both had alcohols as the most dominant scent components, only a few stereotypical scent components were observed in this individual species. An overlap in five of 66 compounds was detected between the two cultivars. Similar results were also observed in I. pallida, two cultivars of which had different terpene and ester dominant scent components, respectively. More interestingly, the highest relative content of esters was detected in I. germanica wild species, however in cultivars, the highest level of alcohols, terpenes and alkanes were detected as well, which indicates that the floral scent profile in I. germanica group has become more complex during the cultivation and domestication process. The related species such as I. pallida and I. pumila which have high contents of terpenes and alcohols might play an important role on the floral scent evolution of I. germanica cultivars group. The high complexity of floral scent components provides a wide range of genetic basis for improving and developing new bearded iris varieties with different types of fragrance in the future.

\subsection{Principle Components Analysis of Floral Scent Components in Bearded Irises}

Principle components analysis (PCA) was performed to simplify the multidimensional dataset based on the scent components profiles (Table S1). The results showed that the contribution rates of PC1-PC5 were $28.197 \%, 16.975 \%, 10.378,8.528 \%$ and $7.463 \%$, respectively, and the cumulative contribution rate reached $71.541 \%$, which indicated that the first five PCs covered most of the scent component information of the investigated materials. As a result, we selected the component with the highest loading value in each of the first five PCs as the main factor, which showed that 1-caryophyllene, linalool, citronellol, methyl cinnamate and $\beta$-cedrene contributed the most to the scent components. Furthermore, as the cumulative contribution rate of the first three PCs reached $55.550 \%$, we created a $3 \mathrm{D}$ loading plot to further explore the influence of each floral scent component on the differentiation of the twenty-seven accessions (Figure 1). The components with higher loading values explained more variance while those with low loading values explained less variance. The five compounds with the highest loading values in each of the first three PCs were extracted as the main scent components which contributed the most to each principal component. It could be seen that besides 1-caryophyllene, linalool, citronellol, methyl cinnamate and $\beta$-cedrene, thujopsene, methyl myristate, linalyl acetate, isosafrole, nerol and geraniol were also the representative floral scent components in the three bearded iris species. 


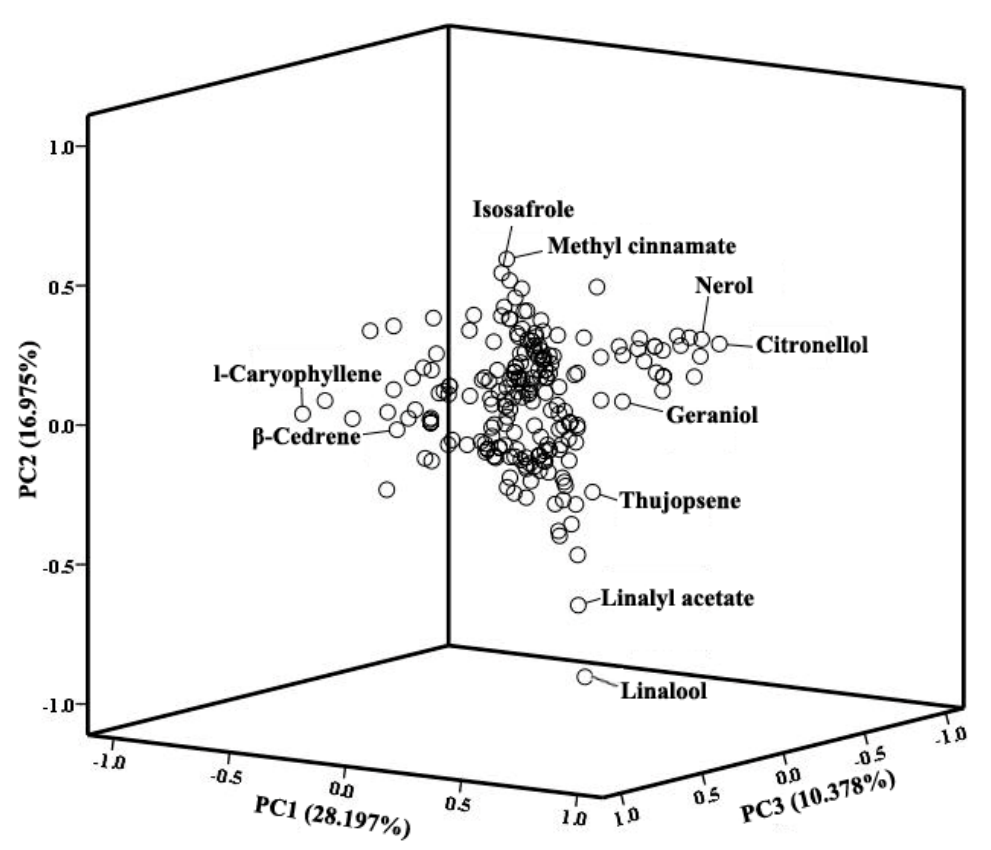

Figure 1. 3D loading plot of Eigenvector load values for 219 floral scent components from PC1, PC2 and PC3.

In addition, we also performed PCA analysis based on the 10 categories of floral scent components in the twenty-seven accessions (Figure 2). The first two PCs accounted for $90.359 \%$ of the total variance. PC1 explained 56.550\%: terpene components were negative, while alcohols and esters were positive. PC2 (33.809\% of the total variance) was positively related to alcohols and terpenes while negatively related to esters. The loading values of terpenes, alcohols and esters were the highest in both PCs indicating that the three categories of scent components contributed the most to the floral scent components in the three bearded iris species.

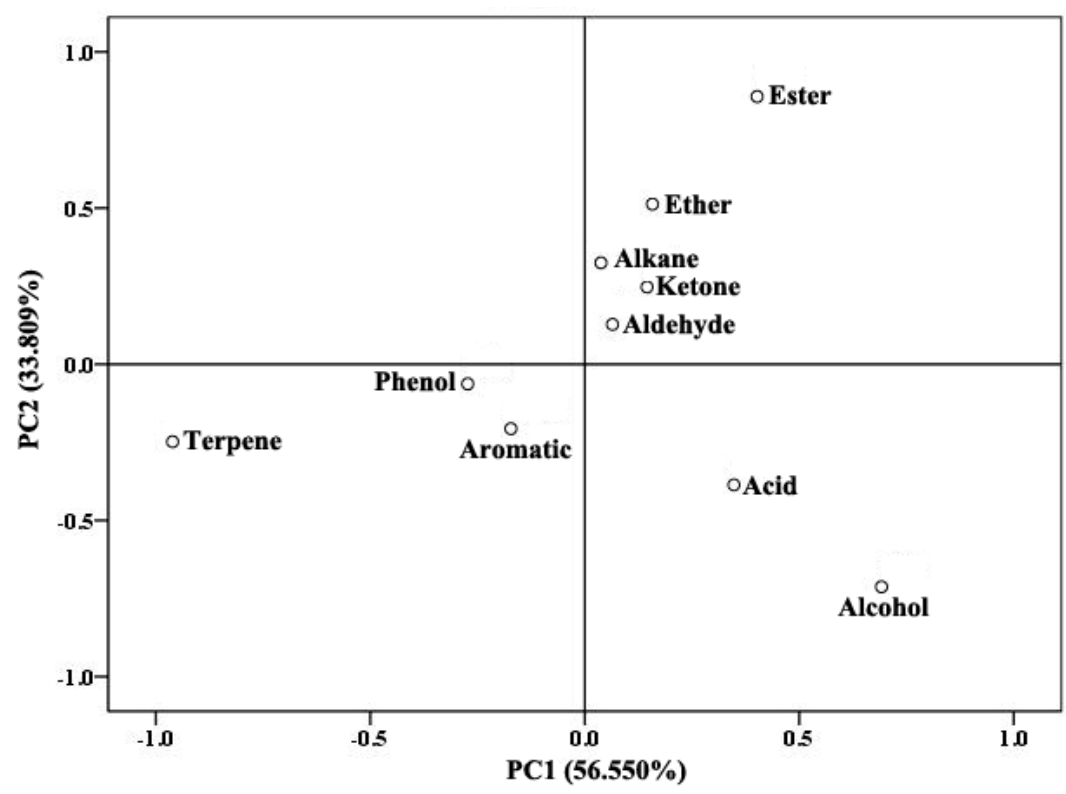

Figure 2. 2D loading plot of eigenvector load values for 10 floral scent component categories from PC1 and PC2.

Bearded irises are not only ornamental plants, but also aromatic plants whose flowers are characterized by violet-like scent [28] and rhizomes constitute important sources of aromatic raw 
materials [29]. During a long drying time (3-5 years), the fats and oils in rhizomes undergo degradation and oxidation and then could release fragrant compounds called irones which could be used for iris essential oil production. Their scent was reported to resemble the one of violet flowers [28]. Besides, in I. germanica and I. pallida, an extract called resinoid with quite different odour (chocolate, woody, leathery and hay scent) could also be obtained from rhizomes, and eight isoflavones were identified in both resinoids [30]. However in our study, little irones and isoflavones were detected in the floral scent indicating that the composition of the floral scent emitted from flowers differs from that of the iris essential oil and resinoids extracted from rhizomes, and the secondary metabolites in iris flowers might be different from those in rhizomes. Similar results were also reported in two beardless species I. pseudacorus and I. kerneriana [31]. Moreover, the main floral volatiles detected in I. pseudacorus and I. kerneriana flowers, e.g. hexadecanoic acid, heptacosane, 6-methyl-5-hepten-2-one in I. pseudacorus and va-kessyl acetate, longipinene, decanoic acid, heptacosane, hexadecanoic acid, 6-methyl-5-hepten-2-one in I. kerneriana [31], were detected little in our study, which might indicate the different floral scent profiles between bearded and beardless irises. In our study, the floral scent volatiles could be classified into 10 categories including terpenes, alkanes, aromatic compounds, esters, alcohols, ketones, aldehydes, ethers, phenols and acids. Terpenes, alcohols, esters were the major components of floral scent according to PCA analysis. Similar classification was also reported in Lilium [7] and Rosa rugosa [8]. Our result provides a detailed evaluation for floral scent profile in bearded iris. However, the floral volatile organic compounds could be divided into terpenoids, phenylpropanoids/benzenoids, fatty acid derivatives, sulfur- and nitrogen-containing compounds based on major biosynthesis pathways [32]. Further investigations need to be carried out on the identification of the biosynthesis pathways in the differentially floral scent phenotypic accessions so as to mine the involved differentially expressed genes and analyze the relevant functions on floral scent regulation.

\subsection{Hierarchical Cluster Analysis of Floral Scent Components in Bearded Irises}

To compare floral scent compositions among the twenty-seven accessions, we performed a hierarchical cluster analysis based on the relative contents of the 219 floral scent components. As is shown in Figure 3, by using Ward's method for between-groups linkage and the squared Euclidean distance between clusters as a proximity measurement, twenty-seven accessions are clustered into six major groups. Eleven accessions including ten I. germanica cultivars and an I. pumila cultivar 'Dash Away' are clustered into Group I with higher relative content of linalool (12.22-34.61\%). Six accessions including five I. germanica cultivars and an I. pallida cultivar 'Albo Variegata' are clustered into Group II with higher relative contents of methyl myristate (0-22.86\%), methyl caprate (0-13.08\%) or citronellyl acetate (0-15.90\%). Group III contains accessions 3 (I. germanica 'Swalli') and 14 (I. germanica 'Abridge Version'), which is with higher relative content of thujopsene $(17.33 \%-21.92 \%)$. Group IV contains accessions 7 (I. germanica 'Forever Blue') and 25 (I. pumila 'Brassie'), which is with higher relative content of citronellol (23.58-33.64\%). Accessions 1 (I. germanica wild species) and 12 (I. germanica 'Spiced Custard') are in Group V with higher relative content of methyl cinnamate (22.59-34.16\%). Finally, accessions 4 (I. germanica 'Casual Elegance'), 5 (I. germanica 'Blessed Again'), 22 (I. germanica 'Amsterdam') and 26 (I. pallida 'Dalmatica') are clustered into Group VI with higher relative content of 1-caryophyllene (24.64\%-52.38\%).

Surprisingly, hierarchical cluster analysis didn't separate the three investigated species. The different accessions of I. pumila and I. pallida were clustering far apart: I. pumila in Groups I and IV and I. pallida Groups II and VI. Generally, the floral scent profile is species-specific, such as in the genus of Magnolia [33] and Antirrhinum [34]. However, certain environmental factors (including pollinators) as well as hybridized introgression could cause chemical change in the floral scent [33,35]. In our study, the three investigated species are all cultivated groups which have occurred wide gene recombination during the long history of selection and breeding. Linalool and linalyl acetate account for $56.60 \%$ of the floral scent in I. pumila 'Dash Away' while citronellol and nerol account for $48.83 \%$ in I. pumila 'Brassie'. 
Thirty-six compounds are detected in I. pallida 'Dalmatica' while fifty-nine in I. pallida 'Albo Variegata'. The large difference of the main floral scent components and composition might be responsible for the cluster results.

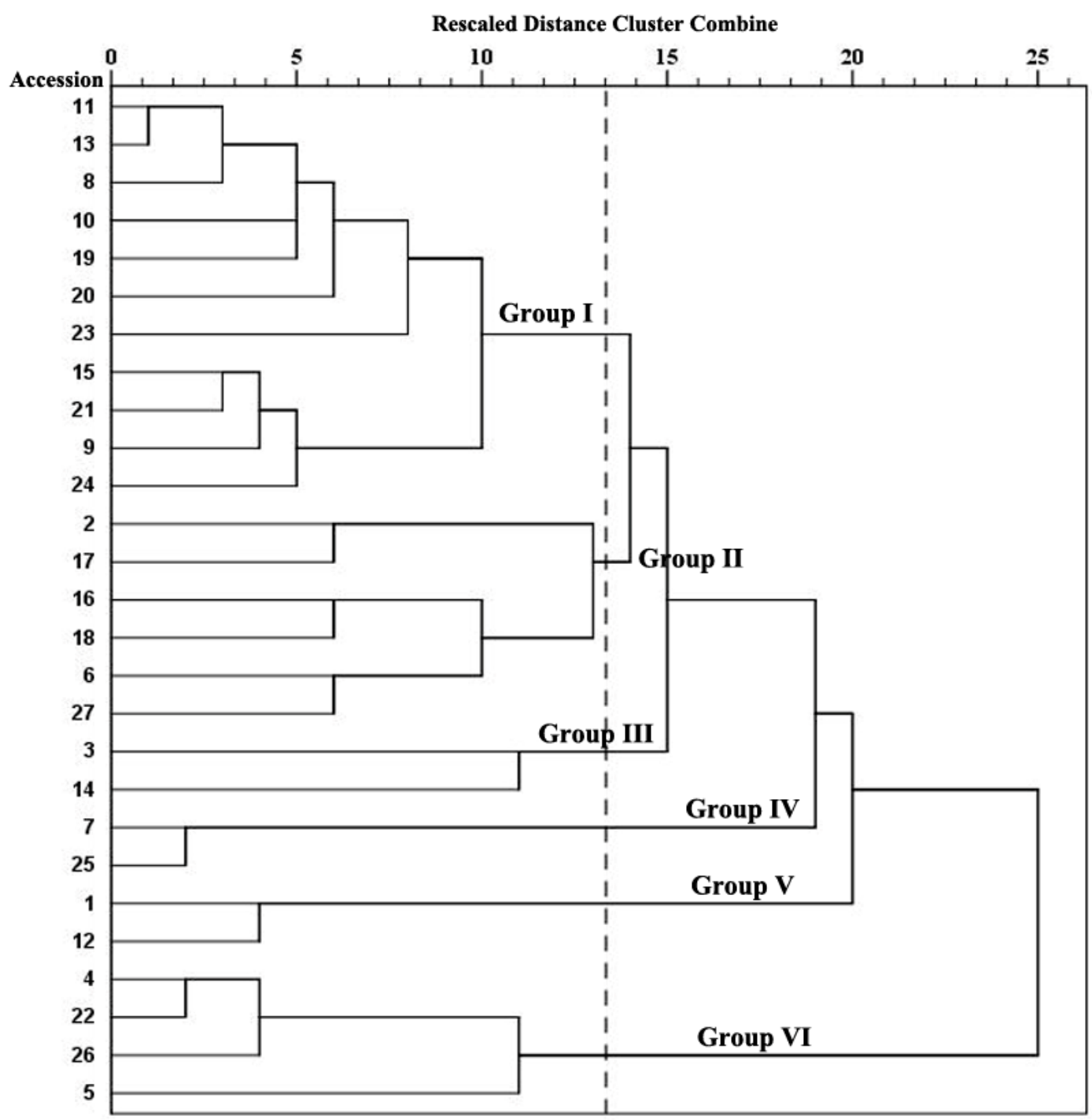

Figure 3. Hierarchical cluster dendrogram of twenty-seven bearded iris accessions.

Floral scent is formed by the interaction of various volatile components. The component with higher scent value (content/olfactory threshold) could be considered as the characteristic floral scent component [36]. According to the result of hierarchical cluster analysis, most of the groups have representative scent components which are with higher relative contents. As previously reported, linalool, citronellyl acetate, thujopsene, citronellol, methyl cinnamate, 1-caryophyllene were responsible for the characteristic odor or fragrance profiles of sweet, lemon fruit-like, woody, rose flower-like, strawberry fruit-like and spicy [37,38], respectively. Therefore, the present study provides a more effective way for the olfactory evaluation and classification of the bearded irises with different floral fragrance, which would be an important theoretical reference for parents selecting and floral fragrance breeding in the future. 


\section{Materials and Methods}

\subsection{Plant Materials}

A total of twenty-seven accessions including the wild species and twenty-two cultivars of I. germanica, two I. pumila cultivars, and two I. pallida cultivars were used in this study (Table 3). All the plant materials were grown under the same fertilizer and water management in the Iris Germplasm Resource Nursery $\left(32^{\circ} 25^{\prime} \mathrm{N} ; 1^{1} 9^{\circ} 23^{\prime} \mathrm{E}\right)$ in Institute of Agricultural Sciences for Lixiahe Region in Jiangsu, China.

Table 3. Species and cultivars of bearded irises used in this study.

\begin{tabular}{cc}
\hline Code & Accession \\
\hline 1 & I. germanica wild species \\
2 & I. germanica 'Crown Princess' \\
3 & I. germanica 'Swahili' \\
4 & I. germanica 'Casual Elegance' \\
5 & I. germanica 'Blessed Again' \\
6 & I. germanica 'Hold and Behold' \\
7 & I. germanica 'Forever Blue' \\
8 & I. germanica 'Crinoline' \\
9 & I. germanica 'Immortality' \\
10 & I. germanica 'Indian chief' \\
11 & I. germanica 'Summer Olympics' \\
12 & I. germanica 'Spiced Custard' \\
13 & I. germanica 'Lenora Pearls' \\
14 & I. germanica 'Abridged Version' \\
15 & I. germanica 'Lent a Williamson' \\
16 & I. germanica 'Rare Edition' \\
17 & I. germanica 'Rajah Brooke' \\
18 & I. germanica 'Superstition' \\
19 & I. germanica 'Splashacata' \\
20 & I. germanica 'I' ve Got Rhythm' \\
21 & I. germanica 'Tulip Festival' \\
22 & I. germanica 'Amsterdam' \\
23 & I. germanica 'Wanda Rezac' \\
24 & I. pumila 'Dash Away' \\
25 & I. pumila 'Brassie' \\
26 & I. pallida 'Dalmatica' \\
27 & I. pallida 'Albo Variegata' \\
\hline
\end{tabular}

\subsection{Sample Collection}

From April to May in 2018, the whole blooming flowers with fall petals fully expanded and the top of standard petals still closed (the second day after flower opening) were collected.

Each cut flower was immediately placed into a $100 \mathrm{~mL}$ capped solid-phase microextraction vial (Figure 4), and then quickly transported to the laboratory for scent collection. All samples were taken within $60 \mathrm{~min}$ before floral scent sampling. These and subsequent procedures were repeated three times for each species and cultivar. 


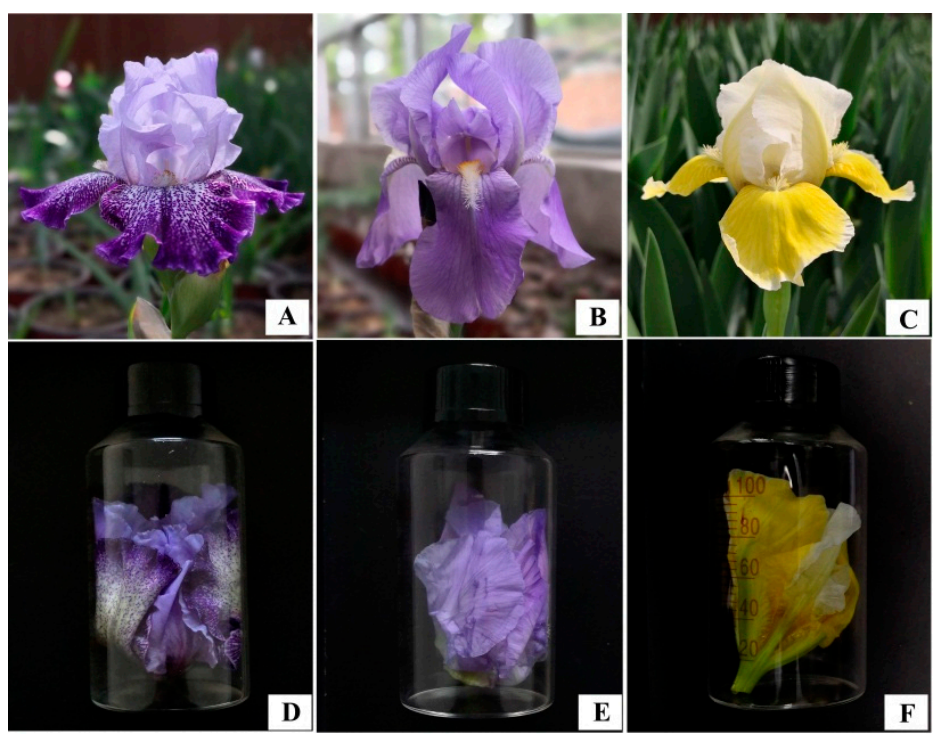

Figure 4. Photos of the representative accession for each of the three bearded iris species while flowering and inside $100 \mathrm{~mL}$ vials used for headspace extractions. (A,D) I. germanica 'Splashacata'; (B,E) I. pallida 'Dalmatica'; (C,F) I. pumila 'Dash Away'.

\subsection{HS-SPME Analysis}

HS-SPME analysis was performed by using a $75 \mu \mathrm{m}$ carboxen-polydimethylsiloxane (CAR-PDMS) SPME fiber equipped with a manual SPME holder (Supelco, Bellefonte, PA, USA). Samples were equilibrated for $20 \mathrm{~min}$ at room temperature before analysis and the SPME fiber was conditioned at the gas chromatographic injection port for $40 \mathrm{~min}$ at $250^{\circ} \mathrm{C}$ before the first volatile collection. After that, the fiber was inserted into the headspace of the capped vial with SPME holder to absorb volatile compounds for $40 \mathrm{~min}$ at $45^{\circ} \mathrm{C}$ (water bath). The empty capped vial was used as the blank control.

\subsection{GC-MS Analysis}

When the extraction was complete, the fibers were withdrawn and inserted into the TRACE ISQ gas chromatography-mass spectrometry (Thermo Company, Waltham, MA, USA), and desorbed at $250{ }^{\circ} \mathrm{C}$ for $2 \mathrm{~min}$. After that, the instrument was activated to collect data. TG-WAXMS column $(60 \mathrm{~m} \times 0.32 \mathrm{~mm} \times 1.0 \mu \mathrm{m})$ was used for chromatographic analysis. The sample volume was $0.2 \mu \mathrm{L}$ and helium (99.99\%) was used as the carrier gas without splitting. The initial oven temperature was maintained at $50{ }^{\circ} \mathrm{C}$ for $1 \mathrm{~min}$ and then raised at $5^{\circ} \mathrm{C} \mathrm{min}-1$ to $120^{\circ} \mathrm{C}$, then at $8{ }^{\circ} \mathrm{C} \mathrm{min}^{-1}$ to $200{ }^{\circ} \mathrm{C}$, and finally at $12^{\circ} \mathrm{C} \mathrm{min}^{-1}$ to $250^{\circ} \mathrm{C}$, maintained for $7 \mathrm{~min}$. The temperatures of the injector, ion source were $250^{\circ} \mathrm{C}$ and $200{ }^{\circ} \mathrm{C}$, respectively. The ionization potential of mass selective detector was $70 \mathrm{eV}$ with the $200 \mathrm{mu}$ emission current and the scan mass range was 45-600 amu.

The volatile compounds formed different chromatographic peaks by the separating of gas chromatography and were qualitatively identified using the TF Xcalibur software by comparing the spectrometric data with those obtained from the NIST 08 mass spectral library and the Wiley library, combined with the manual resolution of mass spectra and confirmed by comparing the Kovat's retention indices and relative reports from the literature [39]. Only results identified with positive and negative matching values of more than 800 (maximum is 1000) were selected and analyzed. All the compounds for each accession were analyzed under the same condition. The relative quantitative analysis was performed through peak normalization procedure. Peak areas were normalized as percentage and used to determine the relative amounts of the volatile compounds. 


\subsection{Statistical Analysis}

The relative contents of all scent compounds emitted from the twenty-seven accessions were subjected to principal components analysis (PCA) by using a covariance matrix to calculate the Eigenvector load values and investigate the major floral scent components utilizing IBM SPSS version 20.0 (IBM, Armonk, NY, USA). Hierarchical cluster analysis was aslo performed to obtain consistent cluster results using Ward's method, and the squared Euclidean distance between clusters was selected as the proximity measurement.

\section{Conclusions}

A total of 219 volatile compounds in floral scent were detected from fully open flowers of twenty-seven accessions in three bearded iris species I. germanica, I. pumila and I. pallida. There is considerable among/within species variation in floral scent components, which provides an extensive genetic basis for scent phenotype improving of bearded irises, especially I. germanica. The compositions of floral scent in bearded irises are different from those of essential oil and resinoid extracted from rhizomes, and also shows great difference compared to the beardless species I. pseudacorus and I. kerneriana. Although hierarchical cluster analysis couldn't separate the three species, our finding that bearded irises could be clustered into different groups which had characteristic odor or fragrance profiles provides a reasonable and effective guidance for the olfactory evaluation and future breeding programs in bearded irises.

Supplementary Materials: The following are available online. Table S1: Loading matrix and Eigenvector values of the first 5 PCs, Figure S1: Total ionic chromatogram of scent components emitted from the flowers of twenty-seven accessions of three bearded iris species.

Author Contributions: Conceived and designed the experiments: F.L., J.B. and Y.Y. Performed the experiments: Y.Y. and F.L. Analyzed the data: Y.Y., Y.S., Y.Z., C.L. and X.C. Contributed reagents/materials/analysis tools: F.L. and J.B. Wrote the paper: Y.Y. and F.L. All authors read and approved the final manuscript.

Acknowledgments: This work was financially supported by Jiangsu Agricultural Science and Technology Innovation Fund [CX (18) 2021], the Earmarked Fund for Jiangsu Agricultural Industry Technology System (JATS [2018] 278), Yangzhou Modern Agriculture Project (YZ2018035) and the Scientific Research Fund of Institute of Agricultural Sciences for Lixiahe Region in Jiangsu [SJ (17) 102].

Conflicts of Interest: The authors declare that they have no conflict of interest.

\section{References}

1. Schiestl, F.P. The Evolution of Floral Scent and Insect Chemical Communication. Ecol. Lett. 2010, 13, 643-656. [CrossRef] [PubMed]

2. Xiang, L.; Chen, L. Adavances in Genetic Engineering of Floral Scent. Sci. Agric. Sin. 2009, 42, 2076-2084.

3. Zhang, Y.; Li, X.; Wang, Y.; Tian, M.; Fan, M. Changes of Aroma Components in Oncidium Sharry Baby in Different Florescence and Flower Parts. Sci. Agric. Sin. 2011, 44, 110-117.

4. Dormont, L.; Delle-Vedove, R.; Bessière, J.-M.; Schatza, B. Floral Scent Emitted by White and Coloured Morphs in Orchids. Phytochemistry 2014, 100, 51-59. [CrossRef] [PubMed]

5. Cao, H.; Li, Z.; Shen, D. GC/MS Fingerprint Analysis of Osmanthus fragrans Lour. in Different Varieties. Acta Hortic. Sin. 2009, 36, 391-398.

6. Li, S.; Chen, L.; Xu, Y.; Wang, L.; Wang, L. Identification of Floral Fragrances in Tree Peony Cultivars by Gas Chromatography-Mass Spectrometry. Sci. Hortic. 2012, 142, 158-165. [CrossRef]

7. Zhang, H.; Hu, Z.; Leng, P.; Wang, W.; Xu, F.; Zhao, J. Qualitative and Quantitative Analysis of Floral Volatile Components from Different Varieties of Lilium spp. Sci. Agric. Sin. 2013, 46, 790-799.

8. Feng, L.-G.; Chen, C.; Sheng, L.-X.; Liu, P.; Tao, J.; Su, J.-L.; Zhao, L.-Y. Comparative Analysis of Headspace Volatiles of Chinese Rosa rugosa. Molecules 2010, 15, 8390-8399. [CrossRef] [PubMed]

9. Karami, A.; Khosh-Khui, M.; Salehi, H.; Saharkhiz, M.J.; Rowshan, V. Headspace Analysis of Floral Scent from Two Distinct Genotypes of Iranian Damask Rose (Rosa damascena Mill.). J. Essent. Oil Bear. Plants 2013, 16, 489-498. [CrossRef] 
10. Zhao, Y.; Pan, H.; Zhang, Q.; Pan, C.; Cai, M. Dynamics of Fragrant Compounds from Prunus mume Flowers. J. Beijing Forest. Univ. 2010, 32, 201-206.

11. Zhou, J.; Ni, D. Changes in Flower Aroma Compounds of Cultivars of Chimonanthus praecox (L.) Link and at Different Stages Relative to Chimonanthus Tea Quality. Acta Hortic. Sin. 2010, 37, 1621-1628.

12. Li, Y.; Ma, H.; Wan, Y.; Li, T.; Liu, X.; Sun, Z.; Li, Z. Volatile Organic Compounds Emissions from Luculia pinceana Flower and Its Changes at Different Stages of Flower Development. Molecules 2016, $21,531$. [CrossRef] [PubMed]

13. Liu, Q.; Sun, G.; Wang, S.; Lin, Q.; Zhang, J.; Li, X. Analysis of the Variation in Scent Components of Hosta Flowers by HS-SPME and GC-MS. Sci. Hortic. 2014, 175, 57-67. [CrossRef]

14. Xu, W.; Cai, M.; Pan, H.; Zhang, Q. Study on Spatial-temporal Variability of Volatile Components in Lagerstroemia indica 'Xiang Xue Yun'. J. Beijing For. Univ. 2017, 39, 85-95.

15. Austin, C. Irises: A Gardener's Encyclopedia; Timber Press: Portland, OR, USA, 2005; p. 23.

16. Wang, G.; Li, D.; Zhang, J.; Xia, Y. Comparison of Cold Tolerance Within 6 Cultivars of Iris germanica. Acta Hortic. Sin. 2014, 41, 773-780.

17. Meyer, C.J.; Peterson, C.A.; Steudle, E. Permeability of Iris germanica's Multiseriate Exodermis to Water, $\mathrm{NaCl}$, and Ethanol. J. Exp. Bot. 2011, 62, 1911-1926. [CrossRef] [PubMed]

18. Guo, J.; Zhang, J.; Sun, G.; Shi, L. Effects of 6-BA Application on Promotion of Offset Formation in Iris germanica. Acta Hortic. Sin. 2007, 34, 461-464.

19. Wang, Y.; Jeknicc, Z.; Ernst, R.C.; Chen, T.H.H. Improved Plant Regeneration from Suspension-Cultured Cells of Iris germanica L. 'Skating Party'. Hortscience 1999, 34, 1271-1276. [CrossRef]

20. Hossein, A.M.; Somayyeh, J.; Rodrigo, B.G. Multivariate Analysis of Morphological Characteristics in Iris germanica Hybrids. Euphytica 2018, 214, 161.

21. Huang, S.Z.; Han, Y.L.; Zhang, Y.G.; Guo, W.M. Breeding Dwarf Plants of Iris germanica L. through Hybridization. J. Nanjing Agric. Univ. 2003, 26, 21-25.

22. Jeknic', Z.; Jeknic', S.; Jevremovic', S.; Subotic', A.; Chen, T.H.H. Alteration of Flower Color in Iris germanica L. 'Fire Bride' through Ectopic Expression of Phytoene Synthase Gene (crtB) from Pantoea agglomerans. Plant Cell Rep. 2014, 33, 1307-1321. [CrossRef] [PubMed]

23. Li, F.; Bao, J.; Sun, Y.; Liu, C.; Ma, H.; Zhang, T.; Chen, X. Biological Effects of ${ }^{60} \mathrm{Co}-\gamma$ Rays Irradiation on the Hybrid Seeds of Iris germanica. J. Nuclear Agric. Sci. 2017, 31, 1469-1474.

24. Sun, H.; Zhang, T.; Fan, Q.; Qi, X.; Zhang, F.; Fang, W.; Jiang, J.; Chen, F.; Chen, S. Identification of Floral Scent in Chrysanthemum Cultivars and Wild Relatives by Gas Chromatography-Mass Spectrometry. Molecules 2015, 20, 5346-5359. [CrossRef] [PubMed]

25. Dobson, H.E.M.; Arroyo, J.; Bergstrom, G.; Groth, I. Interspecific Variation in Floral Fragrances within the Genus Narcissus (Amaryllidaceae). Biochem. Syst. Ecol. 1997, 25, 685-706. [CrossRef]

26. Jürgens, A. Flower Scent Composition in Diurnal Silene Species (Caryophyllaceae): Phylogenetic Constraints or Adaptation to Flower Visitors? Biochem. Syst. Ecol. 2004, 32, 841-859. [CrossRef]

27. Majetic, C.J.; Levin, D.A.; Raguso, R.A. Divergence in Floral Scent Profiles among and within Cultivated Species of Phlox. Sci. Hortic. 2014, 172, 285-291. [CrossRef]

28. Kukula-Koch, W.; Sieniawska, E.; Widelski, J.; Urjin, O.; Głowniak, P.; Skalicka-Woźniak, K. Major Secondary Metabolites of Iris spp. Phytochem. Rev. 2015, 14, 51-80. [CrossRef]

29. Hacibekiroglu, I.; Kolak, U. Antioxidant and Anticholinesterase Constituents from the Petroleum Ether and Chloroform Extracts of Iris suaveolens. Phytother. Res. 2011, 25, 522-529. [CrossRef]

30. Roger, B.; Jeannot, V.; Fernandez, X. Characterisation and Quantification of Flavonoids in Iris germanica L. and Iris pallida Lam. Resinoids from Morocco. Phytochem. Anal. 2012, 23, 450-455. [CrossRef]

31. Basser, K.; Demirci, B.; Orhan, I.E.; Kartal, M.; Sekeroglu, N.; Sener, B. Composition of Volatiles from Three Iris Species of Turkey. J. Essent. Oil Res. 2011, 23, 66-71. [CrossRef]

32. Mühlemann, J.K.; Klempien, A.; Dudareva, N. Floral Volatiles: From Biosynthesis to Function. Plant Cell Environ. 2014, 37, 1936-1949. [CrossRef]

33. Azuma, H.; Thien, L.B.; Kawano, S. Molecular Phylogeny of Magnolia (Magnoliaceae) Inferred from cpDNA Sequences and Evolutionary Divergence of the Floral Scents. J. Plant Res. 1999, 112, 291-306. [CrossRef]

34. Weiss, J.; Mühlemann, J.K.; Ruiz-Hernández, V.; Dudareva, N.; Egea-Cortines, M. Phenotypic Space and Variation of Floral Scent Profiles during Late Flower Development in Antirrhinum. Front. Plant Sci. 2016, 7 , 1903. [CrossRef] [PubMed] 
35. Raguso, R.A.; Schlumpberger, B.O.; Kaczorowski, R.L.; Holtsford, T.P. Phylogenetic Fragrance Patterns in Nicotiana Sections Alatae and Suaveolentes. Phytochemistry 2006, 67, 1931-1942. [CrossRef] [PubMed]

36. Liu, Q.; Sun, G.; Zhang, J.; Li, X. Study on Floral Scent of the Genus Hosta. Sci. Agric. Sin. 2015, 48, 4323-4334.

37. Minh Tu, N.T.; Onishi, Y.; Choi, H.; Kondo, Y.; Bassore, S.M.; Ukeda, H.; Sawamura, M. Characteristic Odor Components of Citrus sphaerocarpa Tanaka (Kabosu) Cold-Pressed Peel Oil. J. Agric. Food Chem. 2002, 50, 2908-2913. [CrossRef]

38. Dou, H. Study on the Change of the Main Biochemistry, Aroma Components, as well as Their Influence on the Flavor of Green Tea Beverages during Storage. Master's Thesis, Huazhong Agricultural University, Wuhan, China, 2007.

39. NIST Standard Reference Database. Available online: http://webbook.nist.gov/chemistry (accessed on 10 April 2019).

Sample Availability: Samples of the compounds are not available from the authors.

(C) 2019 by the authors. Licensee MDPI, Basel, Switzerland. This article is an open access article distributed under the terms and conditions of the Creative Commons Attribution (CC BY) license (http://creativecommons.org/licenses/by/4.0/). 